OPEN ACCESS

Edited by:

Vin-Cent Wu,

National Taiwan University, Taiwan

Reviewed by:

Michael Stowasser,

The University of Queensland,

Australia

John Funder,

Hudson Institute of Medical Research,

Australia

Brasilina Caroccia,

University of Padua, Italy

*Correspondence:

Kazutaka Nanba

knamba@umich.edu

Specialty section:

This article was submitted to Neuroendocrine Science,

a section of the journal

Frontiers in Endocrinology

Received: 21 March 2021 Accepted: 07 June 2021

Published: 29 June 2021

Citation:

Nanba K, Rainey WE and Udager AM (2021) Approaches to Gene Mutation

Analysis Using Formalin-Fixed Paraffin-Embedded Adrenal Tumor Tissue From Patients With Primary Aldosteronism.

Front. Endocrinol. 12:683588. doi: 10.3389/fendo.2021.683588

\section{Approaches to Gene Mutation Analysis Using Formalin-Fixed Paraffin-Embedded Adrenal Tumor Tissue From Patients With Primary Aldosteronism}

\author{
Kazutaka Nanba ${ }^{1,2 *}$, William E. Rainey ${ }^{1,3}$ and Aaron M. Udager ${ }^{4,5,6}$ \\ ${ }^{1}$ Department of Molecular and Integrative Physiology, University of Michigan, Ann Arbor, MI, United States, ${ }^{2}$ Department of \\ Endocrinology and Metabolism, National Hospital Organization Kyoto Medical Center, Kyoto, Japan, ${ }^{3}$ Division of Metabolism, \\ Endocrinology, and Diabetes, Department of Internal Medicine, University of Michigan, Ann Arbor, MI, United States, \\ ${ }^{4}$ Department of Pathology, University of Michigan, Ann Arbor, MI, United States, ${ }^{5}$ Rogel Cancer Center, University of \\ Michigan, Ann Arbor, MI, United States, ${ }^{6}$ Michigan Center for Translational Pathology, University of Michigan, Ann Arbor, \\ MI, United States
}

Aldosterone production is physiologically under the control of circulating potassium and angiotensin II as well as adrenocorticotropic hormone and other secretagogues such as serotonin. The adrenal's capacity to produce aldosterone relies heavily on the expression of a single enzyme, aldosterone synthase (CYP11B2). This enzyme carries out the final reactions in the synthesis of aldosterone and is expressed almost solely in the adrenal zona glomerulosa. From a disease standpoint, primary aldosteronism (PA) is the most common of all adrenal disorders. PA results from renin-independent adrenal expression of CYP11B2 and production of aldosterone. The major causes of PA are adrenal aldosterone-producing adenomas (APA) and adrenal idiopathic hyperaldosteronism. Our understanding of the genetic causes of APA has significantly improved through comprehensive genetic profiling with next-generation sequencing. Whole-exome sequencing has led to the discovery of mutations in six genes that cause reninindependent aldosterone production and thus PA. To facilitate broad-based prospective and retrospective studies of APA, recent technologic advancements have allowed the determination of tumor mutation status using formalin-fixed paraffinembedded (FFPE) tissue sections. This approach has the advantages of providing ready access to archival samples and allowing CYP11B2 immunohistochemistryguided capture of the exact tissue responsible for inappropriate aldosterone synthesis. Herein we review the methods and approaches that facilitate the use of adrenal FFPE material for DNA capture, sequencing, and mutation determination.

Keywords: primary aldosteronism, CYP11B2, somatic mutation, immunohistochemistry, next-generation sequencing 


\section{INTRODUCTION}

Technologic advances in genetic analysis have provided us a better understanding of the molecular pathogenesis of endocrine-related tumors. Aldosterone-producing adenoma (APA) is one of the major subtypes of primary aldosteronism (PA), the most common cause of endocrine-related hypertension. The application of next-generation sequencing (NGS) has resulted in the identification of disease-causing mutations in APA and familial PA. Aldosterone-driver mutations can occur in genes encoding membrane ion channels or pumps (1-9). Thus far, APA have been found with mutations in KCNJ5 (1), ATP1A1 (2, 4), ATP2B3 (2), CACNA1D $(3,4), C A C N A 1 H$ (9), and CLCN2 (8) (aldosterone-driver mutations). These mutations cause excess aldosterone production by raising intracellular calcium levels which leads to enhanced CYP11B2 (aldosterone synthase) expression and renin-independent aldosterone production (10). Like other adrenocortical tumors, activating mutations in CTNNB1 gene (encoding $\beta$-catenin) have also been documented in a subset of APA (11-14) but the mechanism of CTNNB1 mutation activation of aldosterone production remains to be clearly defined. So far more than 90 APA somatic mutations have been reported (Table 1). Of note, only part of the previously reported somatic mutations has been functionally tested so far. To assess the pathologic role of these mutations, it would be ideal to perform cell-based studies for each mutation. In addition to tumor somatic mutations, PA aldosterone production may be regulated by hormones that include adrenocorticotropic hormone, serotonin, or luteinizing hormone (37-44).

Since the development of specific antibodies against human CYP11B2, which is required for aldosterone biosynthesis, CYP11B2 immunohistochemistry (IHC) has played an important role in defining the histopathologic characteristics of adrenals from patients with PA $(45,46)$. CYP11B2 IHC has revealed diversities in the histopathology of adrenals from patients with PA, including APA (CYP11B2-expressing adrenocortical adenoma) and adrenals with small CYP11B2expressing cell nests, called aldosterone-producing cell clusters (APCCs) (45) or aldosterone-producing micronodules (APMs) (47). Advanced sequencing methods combined with CYP11B2 IHC have significantly improved the detection rate of somatic mutations in APA $(14,48,49)$. CYP11B2 IHC-guided targeted NGS has also allowed the detection of aldosterone-driver mutations in APCCs (APMs) using small amounts of DNA (50-52). Herein, we provide an overview of recent advances in the genetic analysis of APA and introduce a streamlined sequencing approach using formalin-fixed paraffin-embedded (FFPE) tumor tissue material.

\section{IMPORTANCE OF CYP11B2 IHC AND TARGETED DNA CAPTURE}

Development of specific antibodies against human CYP11B2 has allowed detection of the source of pathologic aldosterone production in the resected adrenal tissue $(45,46)$. Unique characteristics of adrenals from patient with PA have been documented by CYP11B2 IHC. Importantly, adrenal tumors detected by cross-sectional imaging study are not always the cause of aldosterone excess even when adrenal vein sampling lateralizes autonomous aldosterone production to the tumor side (53). In such cases, APA can be below the detection limit of imaging studies and/or imaging-detected tumors can be nonfunctioning adrenocortical adenomas (CYP11B2-negative tumors by IHC). Cases with multiple APAs within one adrenal have also been documented $(14,48,49,54)$.

Traditionally, DNA and RNA have been isolated from snap frozen tumor pieces obtained during pathologic gross dissection at the time of adrenalectomy. Mutational analysis has subsequently been performed without consideration of CYP11B2 expression prior to sequencing. In the largest mutation prevalence study using this conventional approach, aldosterone-driver somatic mutations were detected in 54\% of 474 adrenal tumors from PA patients (55). Considering the aforementioned diversities in the histology of PA, this approach could negatively affect the accuracy of mutational analysis. As such, we recently developed an advanced molecular profiling method using selective DNA isolation from FFPE sections based on CYP11B2 IHC, followed by NGS $(14,56)$. The step-by-step sequencing method using the CYP11B2 IHC-guided approach is shown in Figure 1. Many laboratories, including ours, use a mouse monoclonal antibody specific for human CYP11B2 that was produced and characterized by Dr. Celso Gomez-Sanchez (46). This antibody is commercially available from Millipore Sigma (MABS1251, RRID: AB_2783793), making it useful for both research and pathologic diagnosis purposes. As is needed for most antibodies, laboratory testing for individual in-house protocols should be done to optimize specificity and sensitivity for CYP11B2 detection. Initial protocol testing is particularly important due to the variable CYP11B2 expression seen between APAs. The scanned slide images of adrenal tumor tissue from a PA patient are shown in Figure 2. The adrenal contains two distinct adrenocortical tumors (an APA and a CYP11B2-negative tumor) which exist close to each other. This example highlights the importance of targeted DNA capture method for accurate mutation analysis. Importantly, past studies demonstrated that no aldosterone-driver mutation was detected in CYP11B2-negative adrenocortical tumors from PA patients $(49,57)$.

Using this CYP11B2 IHC-guided approach, aldosteronedriver mutations have been identified in $88-96 \%$ of APAs (14, $48,49)$. A recent study demonstrated a better mutation detection rate using CYP11B2-IHC guided sequencing (94\%) as compared to the authors' previous use of conventional tumor tissue approaches (71\%) (58).

For the laboratories using traditional material, i.e., DNA/ RNA from macro-dissected snap frozen tumor pieces, confirmation of CYP11B2 mRNA expression by quantitative reverse transcription polymerase chain reaction (RT-qPCR) prior to sequencing could also improve the mutation detection 
TABLE 1 | Previously reported somatic mutations in aldosterone-producing adenomas.

\begin{tabular}{|c|c|c|}
\hline \multirow{2}{*}{$\begin{array}{l}\text { Gene } \\
\text { KCNJ5 }\end{array}$} & \multicolumn{2}{|c|}{ Somatic Mutations } \\
\hline & c.343C>T (p.R115W) (15) & c.445_446insTGG (p.T149delinsMA) (49) \\
\hline & c.376T>C (p.W126R) (16) & c.447_448insATT (p.T149delinsTI) (14) \\
\hline & c.414_425dupGCTITCCTGTTC (p.A139_F142dup) (17) & c.450_451insATG (p.I150_G151insM) (13) \\
\hline & c.420C>G (p.F140L) (14) ${ }^{a}$ & c. $451 G>A(p . G 151 R)(1)$ \\
\hline & c.433_434insCCATTG (p.I144_E145insAl) (13) & c.451G>C (p.G151R) (24) \\
\hline & c.433G $>$ C (p.E145Q) (18) & $($ p.G151_Y152del)* (25) \\
\hline & c.433G>A (p.E145K) (4) & c.457_492dupG_G (p.G153_G164dup) (20) \\
\hline & c.432_439delTGAGACCGinsCA (p.E145_E147delinsK) (19) & c.461T>G (p.F154C) (13) \\
\hline & $\begin{array}{l}\text { c.439G }>\text { C and c.448_449insCAACAACCA } \\
\text { (p.E147Q_T149_I150insTाT) (20) }\end{array}$ & c.467_469delTCA (p.I157del) (26) \\
\hline & c.443C>T (p.T148l) $(21)^{b}$ & c.470_471delinsAA (p.l157K) (13) \\
\hline & c.445_446insGAA (p.T148_T149insR) (22) & $\left(p .1157 \_E 159 d e l\right)^{\star}(25)$ \\
\hline & c.446insAAC (p.T149_I150insT) (23) & c. $472 A>G(p . T 158 A)(27)$ \\
\hline & c.445A>T (p.T149S) (21) & c.503T>G (p.L168R) (1) \\
\hline & & $(\text { p.G184E) })^{*}(25)$ \\
\hline & & c.737A>G (p.E246G) (15) \\
\hline \multirow[t]{9}{*}{ ATP1A1 } & c.295G>A (p.G99R) (16) & c.2874_2882delCTTGAAGA (p.F959_E961del) (29) \\
\hline & c.299_313delTCTCAATGTTACTGT (p.F100_L104del) (2) & c.2877_2882delTGAAGA (p.F959_E961delinsL) (28) \\
\hline & c.304_309delATGTTA (p.M102_L103del) (28) & $\begin{array}{l}\text { c.2878_2895delGAAGAGACAGCCCTGGCTinsGCCCTGGTT } \\
\text { (p.E960_A965delinsALV) (48) }\end{array}$ \\
\hline & c.306_317delGTTACTGTGGAT (p.M102_|106delinsW) (28) & c.2877_2888delTGAAGAGACAGC (p.E960_A963del) (29) \\
\hline & c.308_313delTACTGT (p.L103_L104del) (28) & c.2878_2887delGAAGAGACAGinsT (p.E960_A963delinsS) (4) \\
\hline & c.311T>G (p.L104R) (2) & c.2879_2890delAAGAGACAGCCC (p.E960_L964delinsV) (28) \\
\hline & c.995T>G (p.V332G) (2) & c.2878_2892delGAAGAGACAGCCCTGinsGCCGTG (p.E960_L964delinsAV) (14) \\
\hline & c.2864_2878delTATTGGCCTCTTG (p.1955_E960delinsK) (49) & \\
\hline & $\begin{array}{l}\text { c. } 2867 \_2882 d e l T T G G C C T C T T G A A G A i n s G \text { (p.F956_E961delinsW) } \\
\text { (28) }\end{array}$ & \\
\hline \multirow[t]{8}{*}{ ATP2B3 } & c.367G>C (p.G123R) (30) & c.1273_1278delCTGGTC (p.L425_V426del) (2) \\
\hline & c.1228T>G (p.Y410D) (31) & c.1277_1282delTCGTGG (p.V426_V427del) (2) \\
\hline & $\begin{array}{l}\text { c.1264_1278delGTCACTGTGCTGGTCinsAGCACACTC } \\
\text { (p.V422_V426delinsSTL) (22) }\end{array}$ & c.1276_1287delGTCGTGGCTGTC (p.V426_V429del) (28) \\
\hline & c.1264_1275delGTCACTGTGCTGinsATCACT (p.V422_L425delinsIT) & c.1276_1298insGACA_delTCGTGGCTGTCCCAGAGGGCCT \\
\hline & $(14)$ & (p.V426G_V427Q_A428_L433del) (13) \\
\hline & c.1269_1274delTGTGCT (p.V424_L425del) (32) & c.1279_1284delGTGGCT (p.V427_A428del) (33) \\
\hline & c.1270_1275delGTGCTG (p.V424_L425del) (55) & c.1281_1286delGGCTGT (p.A428_V429del) (34) \\
\hline & c.1272_1277delGCTGGT (p.L425_V426del) (2) & \\
\hline \multirow[t]{20}{*}{ CACNA1D } & c.776T>A (p.V259D) (4) & c.2906C>T (p.S969L) (48) \\
\hline & c.776T>G (p.V259G) (14) & c.2936T>A (p.V979D) (55) \\
\hline & c.926T>C (p.V309A) (49) & c.2943G>C (p.V981N) (55) \\
\hline & c. $1201 \mathrm{C}>\mathrm{G}(\mathrm{p} . \mathrm{V} 401 \mathrm{~L})(28)$ & c. $2968 C>G(p . R 990 G)(49)$ \\
\hline & c.1207G>C [p.G403R (exon8A)] $(3,4)$ & c.2969G>A (p.R990H) (4) \\
\hline & c.1207G>C [p.G403R (exon8B)] $(3)^{\star \star}$ & c.2978G>C (p.R993T) (49) \\
\hline & c.1229C>T (p.S410L) (30) & c.2978G>T (p.R993M) (29) \\
\hline & c.1856G>C (p.R619P) (49) & c.2992_2993GC>AT (p.A998I) (55) \\
\hline & c.1955C>T (p.S652L) (55) & c.2993C>T (p.A998V) (55) \\
\hline & c.1964T>C (p.L655P) (55) & c.3019T>C (p.C1007R) (49) \\
\hline & c.2182G>A (p.V728l) (20) & c.3044T>G (p.I1015S) (49) \\
\hline & c. $2222 A>G(p . Y 741 C)(55)$ & c.3044T>C (p.|1015T) (58) \\
\hline & c.2239T>G (p.F747V) (3) & c.3451G>T (p.V1151F) (55) \\
\hline & c.2239T>C (p.F747L) (4) & c.3452T>C (p.V1151A) (29) \\
\hline & c.2241C>G (p.F747L) (4) & c.3455T>A (p.|11152N) (55) \\
\hline & c.2240T>G (p.F747C) (56) & c.3458T>G (p.V1153G) (35) \\
\hline & c.2240T>C (p.F747S) (29) ${ }^{c}$ & c. $4007 \mathrm{C}>\mathrm{G}$ (p.P1336R) (4) \\
\hline & c.2250C>G (p.I750M) $(3,4)$ & c.4012G>A (p.V1338M) (3) \\
\hline & c.2248A>T (p.1750F) (55) & c.4062G>A (p.M1354l) (4) \\
\hline & c.2261A>G (p.N754S) (29) & \\
\hline CACNA1H & c.4289T>C (p.l1430T) (9) & \\
\hline \multirow[t]{2}{*}{ CLCN2 } & c.71G>A (p.G24D) (8) & \\
\hline & c.64-2_74del (36) & \\
\hline
\end{tabular}

\footnotetext{
${ }^{a-c}$ Associated with another somatic mutation ( ${ }^{a} \mathrm{KCNJ} 5$ p.G151R; ${ }^{b} \mathrm{KCNJ5}$ p.T149S; ${ }^{c} \mathrm{CACNA} 1 \mathrm{D}$ p.N754S). ${ }^{*}$ Base change information was not provided in the original article. For the CACNA1D mutations, amino acid substitutions are described based on the reference sequence NM_001128839 otherwise noted (*NM_000720 for the mutation in exon 8B). Mutations that are covered by the primer sets in Table 2 are highlighted in blue.
} 


\section{STEP 1: Preparation of serial FFPE tissue sections \\ $\checkmark$ \\ STEP 2: CYP11B2 IHC \\ STEP 3: Selective sample collection guided by the CYP11B2 IHC (see Figure 2) \\ FFPE slide preparation \\ STEP 4: DNA isolation from captured FFPE material \\ Use of CYP11B2 IHC to define sources of aldosterone, e.g. APA or aldosterone- producing micronodules \\ Tissue capture and DNA isolation \\ STEP 5: Sanger sequencing of KCNJ5 mutation hotspots \\ STEP 6: Targeted NGS of samples without KCNJ5 hotspot mutation \\ DNA sequence analysis \\ STEP 7: Whole exome sequencing for samples without known aldosterone-driver gene mutations \\ FIGURE 1 | Proposed method for DNA mutation analysis using excised adrenal tissue sections from patients with primary aldosteronism. This approach uses CYP11B2 immunohistochemistry (IHC) to define the source of aldosterone for DNA capture in FFPE tissue sections. Captured DNA is then used for Sanger or gene- targeted deep sequencing to detect known and/or novel drivers of aldosterone production.}

A

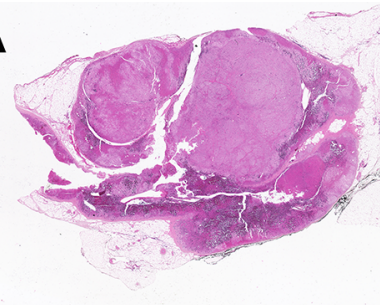

B

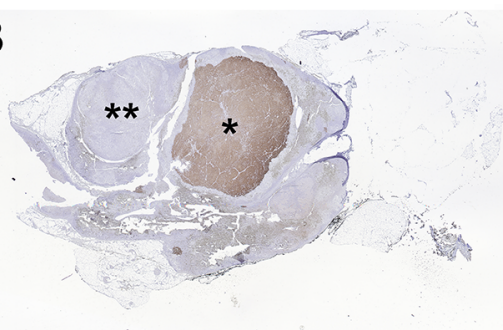

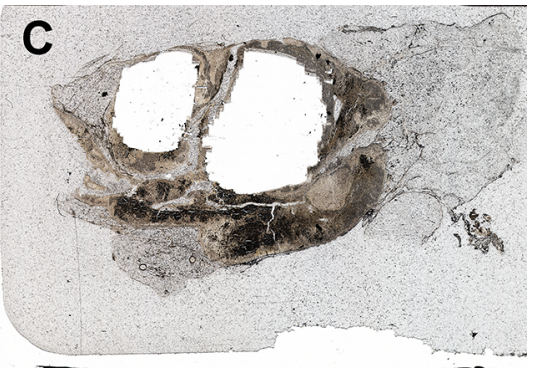

FIGURE 2 | Example of a multinodular adrenal sample from a patient with primary aldosteronism that illustrates the benefit of CYP11B2 IHC-guided DNA capture. (A) Hematoxylin and eosin staining, (B) CYP11B2 immunohistochemistry; *, aldosterone-producing adenoma (APA); ${ }^{*}$, CYP11B2-negative tumor, (C) Post-captured unstained FFPE adrenal tissue section. For DNA isolation, an APA and a CYP11B2-negative tumor were selectively scraped based on the results of CYP11B2 immunohistochemistry.

rate. A proposed method for mutational analysis using banked snap frozen material is shown in Figure 3.

\section{DEFINING SOMATIC MUTATIONS IN ALDOSTERONE-PRODUCING LESIONS}

\section{Sanger Sequencing}

Traditional direct Sanger sequencing has been widely used for the mutational analysis of APA. As new APA-related genes have continuously been identified, it is challenging to perform Sanger sequencing for the screening of multiple genes - particularly for genes like CACNA1D, which have a large coding region with dispersed mutation hotspot areas. Targeted NGS is rapidly becoming the preferred method due to its high sensitivity and ability to utilize small amounts of DNA; however, Sanger sequencing is still an attractive method considering the high per sample cost of NGS. As the prevalence of KCNJ5 hotspot mutations in APA is relatively high, one option to decrease sample throughput is screening for KCNJ5 mutation hotspots 


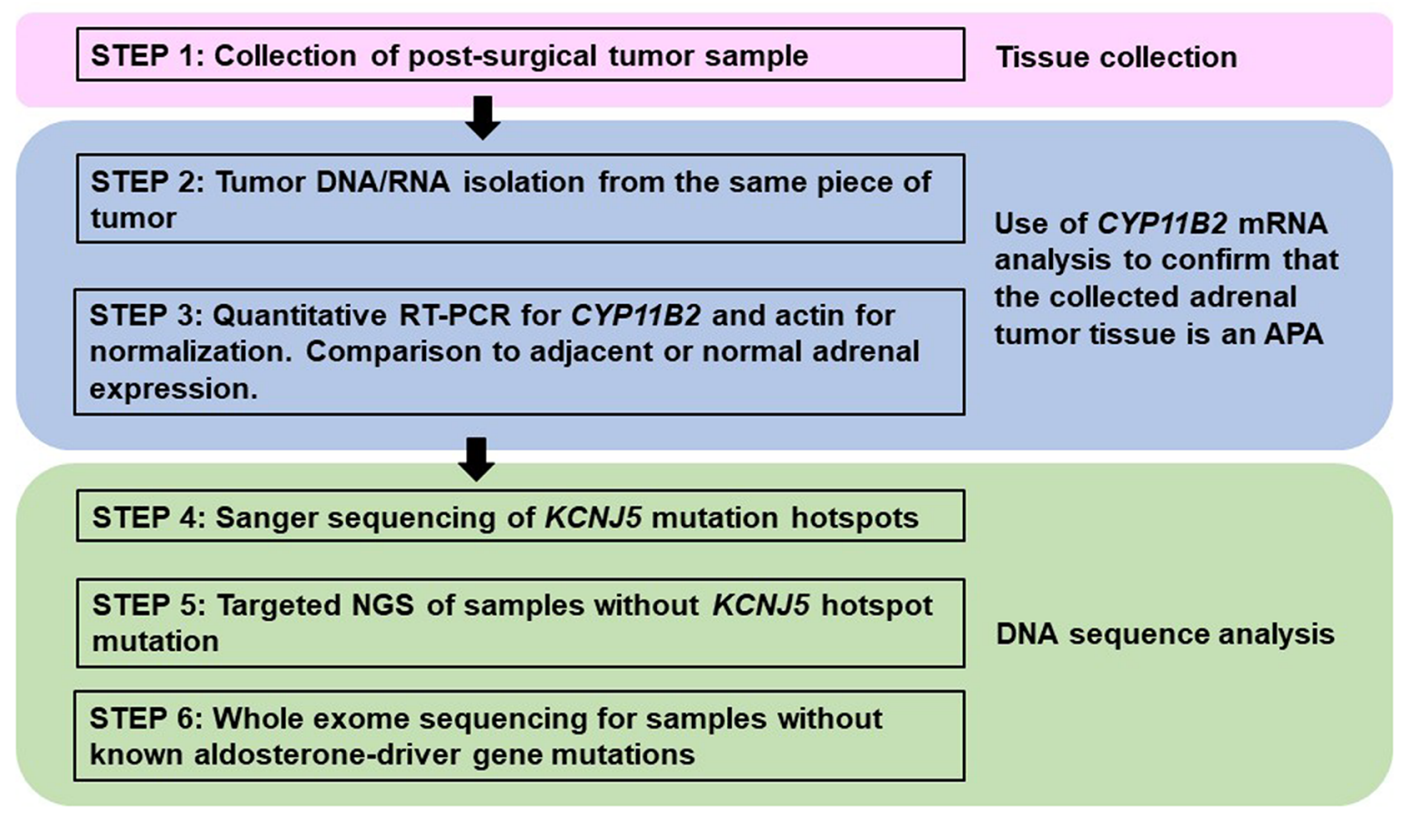

FIGURE 3 | Proposed method for processing fresh or frozen adrenal tumor tissue from patients with primary aldosteronism for mutational analysis. This approach varies from FFPE processing (Figure 1) by the method of tissue collection and the need to use CYP11B2 mRNA detection for confirmation of APA status. However, mutational analysis is similar using Sanger and/or next-generation sequencing to detect known and/or novel drivers of aldosterone production.

using Sanger sequencing, followed by targeted NGS of KCNJ5 mutation-negative samples $(29,48)$ (Figure 1). This approach significantly reduces cost and can also be applied to material isolated from traditional snap frozen tissue (Figure 3). For researchers without available NGS to screen entire coding regions, targeted Sanger sequencing that covers the majority of known aldosterone-driver mutations can be done in a systematic manner. Based on the APA mutation prevalence from our previous study (14), the use of five primer pairs (one for the $K C N J 5$, one for $A T P 1 A 1$, one for $A T P 2 B 3$, and two for CACNA1D, Table 2) appear to be able to identify over $70 \%$ of mutations by direct Sanger sequencing in a Caucasian American cohort. Special consideration is required for primer design when using genomic DNA (gDNA) from FFPE as a template, since FFPE-extracted DNA can be heavily degraded and fragmented.
The authors recommend designing primer sets that target the amplicon size below 250 base pairs (bp) if possible.

\section{Next-Generation Sequencing}

NGS has rapidly become the standard approach for comprehensive molecular profiling of human tumors due to its ability to generate sequence-level genetic data simultaneously for tens, hundreds, or even thousands of genes. Although a variety of NGS methods and platforms exist, there are two broad approaches: amplicon-based and hybridization capture-based (Table 3). Amplicon-based approaches utilize multiplex PCR reactions to amplify genomic regions of interest, while hybridization capture-based methods utilize biotinylated oligonucleotide baits to pull down target regions from pools of sheared gDNA. In general, amplicon-based methods are

TABLE 2 | PCR primer sets for aldosterone-driver mutation hotspots.

\begin{tabular}{|c|c|c|c|c|c|}
\hline Gene & Exon & & Primer Sequences & Amplicon Size (bp) & Reference \\
\hline \multirow[t]{2}{*}{ KCNJ5 } & 2 & Forward & GGACCATGTTGGCGACCAAGAGTG & 211 & $(21)$ \\
\hline & & Reverse & GACAAACATGCACCCCACCATGAAG & & \\
\hline \multirow[t]{2}{*}{ ATP1A1 } & 4 & Forward & АTTAACATCTGCTCGTGCAGCTGAG & 227 & \\
\hline & & Reverse & CCATATGCTGAATTACAGAACTCAC & & \\
\hline \multirow[t]{2}{*}{ ATP2B3 } & 8 & Forward & TGTCTGCCATCACCGTCATCATC & 255 & $(14)$ \\
\hline & & Reverse & CCCAGTITCCGAGTCTGTAAACAG & & \\
\hline \multirow[t]{4}{*}{ CACNA1D } & $8 \mathrm{~A}$ & Forward & CCCACTCCTATGAGACCATC & 190 & \\
\hline & & Reverse & TCTTGGCAACTGTCCTCAGG & & \\
\hline & 16 & Forward & GGTGTGTGGCGTTGCCATTG & 253 & (29) \\
\hline & & Reverse & ААCTGTTGCAGGGCTCCCA & & \\
\hline
\end{tabular}


TABLE 3 | Comparison of NGS approaches for molecular profiling of aldosterone-producing adrenal cortical lesions.

\begin{tabular}{lll}
\hline & Amplicon-based & Hybridization Capture-based \\
\hline Enrichment method & Multiplex PCR & Biotinylated oligonucleotide baits \\
Input DNA & Less & More \\
\# of genomic targets & Fewer & More \\
Experimental time & Less & More \\
Cost per sample & Lower & Higher \\
Application(s) & Targeted sequencing & Targeted sequencing or WES \\
\hline
\end{tabular}

*Depends on depth of sequencing and \# of genomic targets.

WES, whole-exome sequencing

preferred for targeted sequencing of small numbers of genomic regions or when available input DNA for NGS library preparation is very low - particularly for FFPE samples - while hybridization capture-based approaches are favored for analyzing a large number of genomic regions [e.g., wholeexome sequencing (WES)] when ample input DNA is available. These and other differences between the NGS approaches inform how they may be best utilized for molecular profiling of aldosterone-producing lesions using FFPE tissue (Figure 1).

Given the relatively limited number of established aldosteronedriver mutations - coupled with the fact that most of these mutations occur at specific hotspot regions within the affected genes - targeted amplicon-based NGS is ideal for characterizing FFPE APA samples. As mentioned earlier, recent studies utilizing this approach have identified somatic aldosterone-driver mutations in the vast majority of APA. In addition to the ability to interrogate multiple genomic regions simultaneously, one of the important advantages of NGS over Sanger sequencing is improved sensitivity for detecting genetic variants. This is particularly important for detecting somatic mutations in microscopic lesions (i.e., APCC/APM), for which the expected allelic variant fraction may be less than $20 \%$ (depending on the purity of the isolated tissue for sequencing). Application of targeted ampliconbased NGS to APCC in normal adrenal glands and from patients with adrenal idiopathic hyperaldosteronism has identified somatic aldosterone-driver mutations in $34-58 \%$ of these lesions (50-52). For aldosterone-producing lesions that are mutation-negative by targeted amplicon-based NGS, hybridization capture-based WES of CYP11B2 IHC-guided FFPE tissue may identify novel aldosterone-driver mutations $(9,36)$. Finally, despite several clear advantages of NGS-based molecular profiling, application of these approaches to FFPE tissue is potentially limited by FFPEassociated DNA degradation (e.g., increased genomic

\section{REFERENCES}

1. Choi M, Scholl UI, Yue P, Bjorklund P, Zhao B, Nelson-Williams C, et al. K+ Channel Mutations in Adrenal Aldosterone-Producing Adenomas and Hereditary Hypertension. Science (2011) 331:768-72. doi: 10.1126/science. 1198785

2. Beuschlein F, Boulkroun S, Osswald A, Wieland T, Nielsen HN, Lichtenauer UD, et al. Somatic Mutations in ATP1A1 and ATP2B3 Lead to AldosteroneProducing Adenomas and Secondary Hypertension. Nat Genet (2013) 45:440-4, 444e1-2. doi: 10.1038/ng.2550 fragmentation, artifactual nucleotide deamination) and technical issues (e.g., PCR amplification bias, sequencing error). Emerging NGS methods, including the use of unique molecular identifiers (UMI; as known as "molecular barcodes"), and novel NGS technologies may begin to address some of these limitations and will continue to revolutionize genomic characterization of human tumors, including aldosterone-producing lesions.

\section{CONCLUSIONS}

Recent advances in sequencing technology have significantly accelerated PA research to elucidate its molecular pathogenesis. Unique histologic characteristics of adrenals from patients with PA require special attention to tumor CYP11B2 expression for accurate somatic mutation identification. The streamlined approach using CYP11B2 IHC-guided DNA capture combined with NGS appears to be a preferred method for mutational analysis of adrenals from patients with PA. The use of this CYP11B2 IHC-guided sequencing approach in a large prospective cohort will allow us to accurately determine APA mutation prevalence as well as genotype-phenotype correlations.

\section{AUTHOR CONTRIBUTIONS}

$\mathrm{KN}$ and WR conceived the idea of this review article. KN and AU drafted the manuscript. WR reviewed the manuscript and made edits on the contents. All authors contributed to the article and approved the submitted version.

\section{FUNDING}

This work was supported by grants from National Institutes of Diabetes and Digestive and Kidney Disease (DK106618 and DK043140) to WR. KN is supported by the Japan Heart Foundation Research Grant.

\section{ACKNOWLEDGMENTS}

We would like to thank Amy R. Blinder for her editorial assistance. 
Causes Early-Onset Hypertension With Primary Aldosteronism. Elife (2015) 4:e06315. doi: 10.7554/eLife.06315

6. Scholl UI, Stolting G, Schewe J, Thiel A, Tan H, Nelson-Williams C, et al. CLCN2 Chloride Channel Mutations in Familial Hyperaldosteronism Type II. Nat Genet (2018) 50:349-54. doi: 10.1038/s41588-018-0048-5

7. Fernandes-Rosa FL, Daniil G, Orozco IJ, Goppner C, El Zein R, Jain V, et al. A Gain-of-Function Mutation in the CLCN2 Chloride Channel Gene Causes Primary Aldosteronism. Nat Genet (2018) 50:355-61. doi: 10.1038/s41588018-0053-8

8. Dutta RK, Arnesen T, Heie A, Walz M, Alesina P, Soderkvist P, et al. A Somatic Mutation in CLCN2 Identified in a Sporadic AldosteroneProducing Adenoma. Eur J Endocrinol (2019) 181:K37-41. doi: 10.1530/ EJE-19-0377

9. Nanba K, Blinder AR, Rege J, Hattangady NG, Else T, Liu CJ, et al. Somatic CACNA1H Mutation As a Cause of Aldosterone-Producing Adenoma. Hypertension (2020) 75:645-9. doi: 10.1161/HYPERTENSIONAHA.119.14349

10. Fernandes-Rosa FL, Boulkroun S, Zennaro MC. Genetic and Genomic Mechanisms of Primary Aldosteronism. Trends Mol Med (2020) 26:819-32. doi: 10.1016/j.molmed.2020.05.005

11. Akerstrom T, Maharjan R, Sven Willenberg H, Cupisti K, Ip J, Moser A, et al. Activating Mutations in CTNNB1 in Aldosterone Producing Adenomas. Sci Rep (2016) 6:19546. doi: 10.1038/srep19546

12. Wu VC, Wang SM, Chueh SJ, Yang SY, Huang KH, Lin YH, et al. The Prevalence of CTNNB1 Mutations in Primary Aldosteronism and Consequences for Clinical Outcomes. Sci Rep (2017) 7:39121. doi: 10.1038/ srep39121

13. Scholl UI, Healy JM, Thiel A, Fonseca AL, Brown TC, Kunstman JW, et al. Novel Somatic Mutations in Primary Hyperaldosteronism Are Related to the Clinical, Radiological and Pathological Phenotype. Clin Endocrinol (Oxf) (2015) 83:779-89. doi: 10.1111/cen.12873

14. Nanba K, Omata K, Else T, Beck PCC, Nanba AT, Turcu AF, et al. Targeted Molecular Characterization of Aldosterone-Producing Adenomas in White Americans. J Clin Endocrinol Metab (2018) 103:3869-76. doi: 10.1210/ jc.2018-01004

15. Cheng CJ, Sung CC, Wu ST, Lin YC, Sytwu HK, Huang CL, et al. Novel KCNJ5 Mutations in Sporadic Aldosterone-Producing Adenoma Reduce Kir3.4 Membrane Abundance. J Clin Endocrinol Metab (2015) 100(1): E155-63.

16. Williams TA, Monticone S, Schack VR, Stindl J, Burrello J, Buffolo F, et al. Somatic ATP1A1, ATP2B3, and KCNJ5 Mutations in Aldosterone-Producing Adenomas. Hypertension (2014) 63(1):188-95.

17. Hardege I, Xu S, Gordon RD, Thompson AJ, Figg N, Stowasser M, et al. Novel Insertion Mutation in KCNJ5 Channel Produces Constitutive Aldosterone Release From H295R Cell. Mol Endocrinol (2015) 29(10):1522-30.

18. Akerstrom T, Crona J, Delgado Verdugo A, Starker LF, Cupisti K, Willenberg HS, et al. Comprehensive Re-Sequencing of Adrenal Aldosterone Producing Lesions Reveal Three Somatic Mutations Near the KCNJ5 Potassium Channel Selectivity Filter. PloS One (2012) 7(7):e41926.

19. Zheng FF, Zhu LM, Zhou WL, Zhang Y, Li MY, Zhu YC, et al. A Novel Somatic Mutation 145-147deleteinsk in KCNJ5 Increases Aldosterone Production. J Hum Hypertens (2017) 31(11):756-9.

20. Wang B, Li X, Zhang X, Ma X, Chen L, Zhang Y, et al. Prevalence and Characterization of Somatic Mutations in Chinese Aldosterone-Producing Adenoma Patients. Med (Baltimore) (2015) 94(16):e708.

21. Nanba K, Omata K, Tomlins SA, Giordano TJ, Hammer GD, Rainey WE, et al. Double Adrenocortical Adenomas Harboring Independent KCNJ5 and PRKACA Somatic Mutations. Eur J Endocrinol (2016) 175(2):K1-6.

22. Zheng FF, Zhu LM, Nie AF, Li XY, Lin JR, Zhang K, et al. Clinical Characteristics of Somatic Mutations in Chinese Patients With Aldosterone-Producing Adenoma. Hypertension (2015) 65(3):622-8.

23. Kuppusamy M, Caroccia B, Stindl J, Bandulik S, Lenzini L, Gioco F, et al. A Novel KCNJ5-Inst149 Somatic Mutation Close to, But Outside, the Selectivity Filter Causes Resistant Hypertension by Loss of Selectivity for Potassium. J Clin Endocrinol Metab (2014) 99(9):E1765-73.

24. Taguchi R, Yamada M, Nakajima Y, Satoh T, Hashimoto K, Shibusawa N, et al. Expression and Mutations of KCNJ5 mRNA in Japanese Patients With Aldosterone-Producing Adenomas. J Clin Endocrinol Metab (2012) 97 (4):1311-9.
25. Kitamoto T, Omura M, Suematsu S, Saito J, Nishikawa T. KCNJ5 Mutation as a Predictor for Resolution of Hypertension After Surgical Treatment of Aldosterone-Producing Adenoma. J Hypertens (2018) 36(3):619-27.

26. Azizan EA, Lam BY, Newhouse SJ, Zhou J, Kuc RE, Clarke J, et al. Microarray, qPCR, and KCNJ5 Sequencing of Aldosterone-Producing Adenomas Reveal Differences in Genotype and Phenotype Between Zona Glomerulosa- and Zona Fasciculata-Like Tumors. J Clin Endocrinol Metab (2012) 97(5):E819-29.

27. Mulatero P, Tauber P, Zennaro MC, Monticone S, Lang K, Beuschlein F, et al. KCNJ5 Mutations in European Families With Nonglucocorticoid Remediable Familial Hyperaldosteronism. Hypertension (2012) 59(2):235-40.

28. Akerstrom T, Willenberg HS, Cupisti K, Ip J, Backman S, Moser A, et al. Novel Somatic Mutations and Distinct Molecular Signature in AldosteroneProducing Adenomas. Endocr Relat Cancer (2015) 22(5):735-44.

29. Guo Z, Nanba K, Udager A, McWhinney BC, Ungerer JPJ, Wolley M, et al. Biochemical, Histopathological, and Genetic Characterization of PostureResponsive and Unresponsive APA. J Clin Endocrinol Metab (2020) 105(9): e3224-35.

30. Backman S, Akerstrom T, Maharjan R, Cupisti K, Willenberg HS, Hellman P, et al. RNA Sequencing Provides Novel Insights Into the Transcriptome of Aldosterone Producing Adenoma. Sci Rep (2019) 9(1):6269.

31. Wu VC, Huang KH, Peng KY, Tsai YC, Wu CH, Wang SM, et al. Prevalence and Clinical Correlates of Somatic Mutation in Aldosterone Producing Adenoma-Taiwanese Population. Sci Rep (2015) 5:11396.

32. Murakami M, Yoshimoto T, Minami I, Bouchi R, Tsuchiya K, Hashimoto K, et al. A Novel Somatic Deletion Mutation of ATP2B3 in AldosteroneProducing Adenom. Endocr Pathol (2015) 26(4):328-33.

33. Kitamoto T, Suematsu S, Yamazaki Y, Nakamura Y, Sasano H, Matsuzawa Y, et al. Clinical and Steroidogenic Characteristics of Aldosterone-Producing Adenomas With ATPase or CACNA1D Gene Mutation. J Clin Endocrinol Metab (2016) 101(2):494-503.

34. Dutta RK, Welander J, Brauckhoff M, Walz M, Alesina P, Arnesen T, et al. Complementary Somatic Mutations of KCNJ5, ATP1A1, and ATP2B3 in Sporadic Aldosterone Producing Adrenal Adenomas. Endocr Relat Cancer (2014) 21(1):L1-4.

35. Tan GC, Negro G, Pinggera A, Tizen Laim NMS, Mohamed Rose I, Ceral J, et al. Aldosterone-Producing Adenomas: Histopathology-Genotype Correlation and Identification of a Novel CACNA1D Mutatio. Hypertension (2017) 70(1):129-36.

36. Rege J, Nanba K, Blinder AR, Plaska S, Udager AM, Vats P, et al. Identification of Somatic Mutations in CLCN2 in Aldosterone-Producing Adenomas. J Endocr Soc (2020) 4:bvaa123. doi: 10.1210/jendso/bvaa123

37. Kem DC, Weinberger MH, Higgins JR, Kramer NJ, Gomez-Sanchez C, Holland OB. Plasma Aldosterone Response to ACTH in Primary Aldosteronism and in Patients With Low Renin Hypertension. J Clin Endocrinol Metab (1978) 46:552-60. doi: 10.1210/jcem-46-4-552

38. Lefebvre H, Cartier D, Duparc C, Lihrmann I, Contesse V, Delarue C, et al. Characterization of Serotonin(4) Receptors in Adrenocortical AldosteroneProducing Adenomas: In Vivo and In Vitro Studies. J Clin Endocrinol Metab (2002) 87:1211-6. doi: 10.1210/jcem.87.3.8327

39. Zwermann O, Suttmann Y, Bidlingmaier M, Beuschlein F, Reincke M. Screening for Membrane Hormone Receptor Expression in Primary Aldosteronism. Eur J Endocrinol (2009) 160:443-51. doi: 10.1530/EJE-08-0711

40. Albiger NM, Sartorato P, Mariniello B, Iacobone M, Finco I, Fassina A, et al. A Case of Primary Aldosteronism in Pregnancy: Do LH and GNRH Receptors Have a Potential Role in Regulating Aldosterone Secretion? Eur J Endocrinol (2011) 164:405-12. doi: 10.1530/EJE-10-0879

41. Lopez AG, Duparc C, Naccache A, Castanet M, Lefebvre H, Louiset E. Role of Mast Cells in the Control of Aldosterone Secretion. Horm Metab Res (2020) 52:412-20. doi: 10.1055/a-1119-1063

42. Sonoyama T, Sone M, Miyashita K, Tamura N, Yamahara K, Park K, et al. Significance of Adrenocorticotropin Stimulation Test in the Diagnosis of an Aldosterone-Producing Adenoma. J Clin Endocrinol Metab (2011) 96:2771-8. doi: 10.1210/jc.2011-0573

43. El Ghorayeb N, Bourdeau I, Lacroix A. Role of ACTH and Other Hormones in the Regulation of Aldosterone Production in Primary Aldosteronism. Front Endocrinol (Lausanne) (2016) 7:72. doi: 10.3389/fendo.2016.00072

44. St-Jean M, Bourdeau I, Martin M, Lacroix A. Aldosterone is Aberrantly Regulated by Various Stimuli in a High Proportion of Patients With Primary 
Aldosteronism. J Clin Endocrinol Metab (2021) 106:e45-60. doi: 10.1210/ clinem/dgaa703

45. Nishimoto K, Nakagawa K, Li D, Kosaka T, Oya M, Mikami S, et al. Adrenocortical Zonation in Humans Under Normal and Pathological Conditions. J Clin Endocrinol Metab (2010) 95:2296-305. doi: 10.1210/jc.2009-2010

46. Gomez-Sanchez CE, Qi X, Velarde-Miranda C, Plonczynski MW, Parker CR, Rainey W, et al. Development of Monoclonal Antibodies Against Human CYP11B1 and CYP11B2. Mol Cell Endocrinol (2014) 383:111-7. doi: 10.1016/ j.mce.2013.11.022

47. Williams TA, Gomez-Sanchez CE, Rainey WE, Giordano TJ, Lam AK, Marker A, et al. International Histopathology Consensus for Unilateral Primary Aldosteronism. J Clin Endocrinol Metab (2021) 106:42-54. doi: 10.1210/ clinem/dgaa484

48. Nanba K, Yamazaki Y, Bick N, Onodera K, Tezuka Y, Omata K, et al. Prevalence of Somatic Mutations in Aldosterone-Producing Adenomas in Japanese Patients. J Clin Endocrinol Metab (2020) 105:e4066-73. doi: 10.1210/ clinem/dgaa595

49. Nanba K, Omata K, Gomez-Sanchez CE, Stratakis CA, Demidowich AP, Suzuki $\mathrm{M}$, et al. Genetic Characteristics of Aldosterone-Producing Adenomas in Blacks. Hypertension (2019) 73:885-92. doi: 10.1161/HYPERTENSIONAHA.118.12070

50. Nishimoto K, Tomlins SA, Kuick R, Cani AK, Giordano TJ, Hovelson DH, et al. Aldosterone-Stimulating Somatic Gene Mutations Are Common in Normal Adrenal Glands. Proc Natl Acad Sci USA (2015) 112:E4591-9. doi: 10.1073/pnas.1505529112

51. Omata K, Anand SK, Hovelson DH, Liu CJ, Yamazaki Y, Nakamura Y, et al. Aldosterone-Producing Cell Clusters Frequently Harbor Somatic Mutations and Accumulate With Age in Normal Adrenals. J Endocr Soc (2017) 1:787-99. doi: 10.1210/js.2017-00134

52. Omata K, Satoh F, Morimoto R, Ito S, Yamazaki Y, Nakamura Y, et al. Cellular and Genetic Causes of Idiopathic Hyperaldosteronism. Hypertension (2018) 72:874-80. doi: 10.1161/HYPERTENSIONAHA.118.11086

53. Nanba AT, Nanba K, Byrd JB, Shields JJ, Giordano TJ, Miller BS, et al. Discordance Between Imaging and Immunohistochemistry in Unilateral
Primary Aldosteronism. Clin Endocrinol (Oxf) (2017) 87:665-72. doi: 10.1111/cen.13442

54. Fernandes-Rosa FL, Giscos-Douriez I, Amar L, Gomez-Sanchez CE, Meatchi T, Boulkroun S, et al. Different Somatic Mutations in Multinodular Adrenals With Aldosterone-Producing Adenoma. Hypertension (2015) 66:1014-22. doi: 10.1161/HYPERTENSIONAHA.115.05993

55. Fernandes-Rosa FL, Williams TA, Riester A, Steichen O, Beuschlein F, Boulkroun S, et al. Genetic Spectrum and Clinical Correlates of Somatic Mutations in Aldosterone-Producing Adenoma. Hypertension (2014) 64:35461. doi: 10.1161/HYPERTENSIONAHA.114.03419

56. Nanba K, Chen AX, Omata K, Vinco M, Giordano TJ, Else T, et al. Molecular Heterogeneity in Aldosterone-Producing Adenomas. J Clin Endocrinol Metab (2016) 101:999-1007. doi: 10.1210/jc.2015-3239

57. Dekkers T, ter Meer M, Lenders JW, Hermus AR, Schultze Kool L, Langenhuijsen JF, et al. Adrenal Nodularity and Somatic Mutations in Primary Aldosteronism: One Node is the Culprit? J Clin Endocrinol Metab (2014) 99:E1341-51. doi: 10.1210/jc.2013-4255

58. De Sousa K, Boulkroun S, Baron S, Nanba K, Wack M, Rainey WE, et al. Genetic, Cellular, and Molecular Heterogeneity in Adrenals With Aldosterone-Producing Adenoma. Hypertension (2020) 75:1034-44. doi: 10.1161/HYPERTENSIONAHA.119.14177

Conflict of Interest: The authors declare that the research was conducted in the absence of any commercial or financial relationships that could be construed as a potential conflict of interest.

Copyright (c) 2021 Nanba, Rainey and Udager. This is an open-access article distributed under the terms of the Creative Commons Attribution License (CC BY). The use, distribution or reproduction in other forums is permitted, provided the original author $(s)$ and the copyright owner(s) are credited and that the original publication in this journal is cited, in accordance with accepted academic practice. No use, distribution or reproduction is permitted which does not comply with these terms. 